\title{
Prevalence of malnutrition in nursing and care homes in Walsall
}

\author{
R. Ragubeer ${ }^{1}$ and E. Jerrams ${ }^{2}$ \\ ${ }^{1}$ Walsall Healthcare NHS Trust, Walsall WS2 9PS, UK and ${ }^{2}$ Nutricia Clinical Care, Wiltshire BA14 OXQ, UK
}

Malnutrition is common among older people in nursing and care homes. It is frequently unrecognised and is often under-treated in the $\mathrm{UK}^{(2)}$. Malnutrition costs an estimated $£ 13$ billion in the $\mathrm{UK}^{(3)}$. It is recommended that all patients admitted to a nursing or care home, be screened for malnutrition $^{(2)}$. Despite this, there remains little information on the prevalence of malnutrition in this population. Malnutrition has a negative impact on a patient's health, and in turn this has cost implications for the NHS. Therefore identifying and appropriately treating malnutrition can avoid knock-on healthcare complications and inflated costs.

The aim of this study was to establish the prevalence of malnutrition in older people in nursing and care homes using the 'Malnutrition Universal Screening Tool'(MUST) ${ }^{(1,2,3)}$. The study also aimed to determine how many residents were appropriately prescribed oral nutritional supplements (ONS), and by whom.

630 residents (mean age 82; 72\% female) from 17 nursing, care, residential and mental health homes (5 nursing homes, 5 care homes, 6 residential homes and 1 mental health centre) were screened for malnutrition using 'MUST' from January 2010 to June 2011. The screening was carried out by the clinical community dietitian and Nutricia Nurse, as well as staff trained on the use of MUST.

$58 \%$ (364 residents) were screened as being low risk, $17 \%$ (105 residents) were screened as medium risk and $24 \%$ (150 residents) were screened as high risk. The mean BMI for each risk group was as follows: low risk $\left(25.9 \mathrm{~kg} / \mathrm{m}^{2}\right)$, medium risk $\left(20.1 \mathrm{~kg} / \mathrm{m}^{2}\right)$ and high risk $\left(17.5 \mathrm{~kg} / \mathrm{m}^{2}\right)$. (Please note: 4 residents were not screened as they had just been admitted and no previous data was available, 2 residents were admitted to hospital, 5 refused to be weighed and 1 resident was palliative).

\begin{tabular}{lccr}
\multicolumn{4}{l}{ Table 1. Percentage of screened residents receiving supplements and the source of the prescription } \\
\hline Low risk & Medium risk & High risk \\
\hline Hospital & $5(0.8 \%)$ & $0(0 \%)$ & $9(1.5 \%)$ \\
GP & $10(1.6 \%)$ & $12(2.0 \%)$ & $15(2.4 \%)$ \\
Dietitian & $10(1.6 \%)$ & $9(1.5 \%)$ & $32(5.2 \%)$ \\
\hline
\end{tabular}

From Table 1 , of the $\sim 17 \%$ of residents in the care homes taking supplements, only $\sim 8 \%$ were being monitored and reviewed by a dietitian. 15 low risk and 12 medium risk residents had been prescribed ONS, either when discharged from hospital and from the GP. These residents were not being monitored. The 10 low risk and 9 medium risk residents known to the dietitian, were able to stop the ONS prescribed. After the 'MUST' screening, these 46 residents who were on ONS were able to stop. Appropriate dietary advice was given to the staff on management of these residents, and also to re-screen according to care plans.

Malnutrition is common in the nursing, care and residential homes studied i.e. $\sim 41 \%$ of residents $(17 \%$ medium risk and $24 \%$ high risk). $13 \%$ of residents on ONS were at risk of malnutrition according to 'MUST' (3.5\% medium risk, $\sim 9.0 \%$ high risk). Most of the residents with malnutrition did not receive ONS or dietetic input for oral nutritional support. Residents in the homes studied were inappropriately prescribed ONS without further review (Table 1): $2.4 \%$ of low risk residents on ONS prescribed by either the hospital or GP, $2.0 \%$ of medium risk residents on ONS prescribed by the GP. Many of the residents in the mental health centre were found to be overweight or obese, and they were referred to the Lifestyle Services.

This study shows that malnutrition is common (41\%) across nursing, care and residential home settings. Without continued staff training and resident screening using 'MUST', malnutrition would have been unrecognised and untreated. The study also showed that identifying and appropriately treating malnutrition could potentially reduce health care costs, as well as inappropriate prescribing of ONS.

1. Elia M (2003) The 'MUST' report. Nutritional screening for adults: a multidisciplinary responsibility. Redditch, UK, BAPEN.

2. NICE Guideline (2006) Nutrition support in adults. Clinical guideline 32.

3. www.bapen.org.uk 